\title{
Role of 7-Joint Ultrasonic Score in Predicting the Prognosis of Rheumatoid Arthritis
}

\author{
Xiao-Pan Zou (iD) ${ }^{1}$, Jin-Mei Zou (iD ${ }^{2,}{ }^{*}$, Dao-Ning Guo (iD) ${ }^{1}$, Jia-Gao Qi (iD ${ }^{1}$, Yue Li (iD ${ }^{1}$ and Gang Chen (iD) ${ }^{3}$ \\ ${ }^{1}$ Department of Ultrasound, Mianyang Central Hospital, Mianyang, China \\ ${ }^{2}$ Department of Rheumatology and Immunology, Mianyang Central Hospital, Mianyang, China \\ ${ }^{3}$ Department of Medical Laboratory, Mianyang Central Hospital, Mianyang, China \\ "Corresponding author: Department of Rheumatology and Immunology, Mianyang Central Hospital, No. 12, Fucheng District, Mianyang, China. Tel: +86-13981154236, Email: \\ letter_zjm@163.com
}

Received 2020 March 23; Revised 2020 July 28; Accepted 2020 August 03.

\begin{abstract}
Background: There is a higher possibility for rheumatoid arthritis (RA) recurrence if patients have subclinical synovitis. Sevenjoint ultrasonic score (US7 score) allows an accurate detection of subclinical synovitis, but its predictive role in the recurrence of RA patients in remission is uncertain.

Objectives: The goal of this study was to explore the role of US7 score in predicting the recurrence of RA patients with disease in clinical remission.

Patients and Methods: Totally, 186 RA patients in clinical remission were recruited and their demographic and clinicobiologic characteristics were collected. The US7 score and disease activity score in 28 joints (DAS28) were recorded as baseline indicators for a 1-year follow-up. Patients were divided into the recurrence group and non-recurrence group according to their recurrent results. The clinical indicators and US7 scores before the follow-up were compared and analyzed between the two groups. Kaplan-Meier and univariate COX regression analysis were used to analyze the effect of US7 score on the recurrence of RA. Receiver-operating characteristics (ROC) curves were established to evaluate the predictive accuracy of DAS 28 and US7 score for the outcome of RA.

Results: Of the 186 RA patients, 55.9\% received conventional disease-modifying antirheumatic drugs (cDMARDs) and $44.1 \%$ received biologic disease-modifying antirheumatic drugs (bDMARDs). Totally, 60 patients (32.3\%) suffered from recurrence and were included in the recurrence group, and the remaining 126 patients were included in the non-recurrence group. The recurrence rate of patients without subclinical synovitis (10.26\%) was lower than that of patients with subclinical synovitis $\left(48.15 \%, \chi^{2}=27.556, \mathrm{P}<0.001\right)$. US7 score was associated with an increased risk of recurrence in RA patients after successful treatment $(\mathrm{P}<0.001$, hazard ratio $=1.363$, $95 \% \mathrm{CI}=1.247-1.488)$. The area under the curve of the prediction model with combined US7 score and DAS 28 was higher (0.904) compared to that of US7 score or DAS $28(\mathrm{P}<0.05)$.

Conclusion: The US7 score is capable of predicting the recurrence of RA patients because of its advantages in identifying subclinical synovitis. The combined model of DAS28 and US7 score was accurate for predicting the recurrence of RA patients in clinical remission.
\end{abstract}

Keywords: 7-Joint Ultrasonic Score, Disease Activity Score in 28 Joints, Rheumatoid Arthritis, Ultrasound, Clinical Remission, Recurrence

\section{Background}

The disease activity of rheumatoid arthritis (RA) is routinely evaluated by disease activity score in 28 joints (DAS28) (1). The goal of RA treatment is to maintain the disease activity in clinical remission (Disease Activity Score [DAS]28 $\leq 2.6$ ) because a complete cure is difficult to achieve (2). However, according to a study performed by Peluso et al. (3), about $47.1 \%$ of RA patients would recur after remission. It will result in bone erosion and disability if the recurrence is not controlled in time (4). Nowa- days, the synovial hyperplasia could be easily observed by grayscale ultrasound (GSUS), and the pathogenic vascularization could be evaluated by color Doppler ultrasound (CDUS) (5). Some studies have reported that synovial inflammation is still detected in patients with RA in clinical remission (6-8), which is called subclinical synovitis. RA patients with disease in remission are more likely to recur if the subclinical synovitis exists (9).

Seven-joint ultrasonic score (US7 score) proposed by Backhaus et al. (10) is currently the most convenient and economical ultrasonic examination for RA. Recent reports 
showed that the US7 score was positively correlated with DAS28, suggesting that it could effectively assess the disease activity of RA as well $(11,12)$. It is worth noting that the US7 score allows a more accurate assessment of subclinical synovitis because it can effectively evaluate the synovial inflammatory condition, which would lead to a more accurate prediction of the prognosis of RA patients. However, few studies explored the role of US7 score in predicting the prognosis and reducing the recurrence rate of RA patients with disease in clinical remission.

\section{Objectives}

The aim of the present study was to evaluate the efficacy of RA treatment by the US7 score, and to explore its predictive role in the prognosis of RA patients with disease in remission, especially those with subclinical synovitis.

\section{Patients and Methods}

\subsection{Study Participants}

This was a longitudinal observational study in which we prospectively followed a cohort of RA patients. Ethical approval for the study was obtained from the ethics committee of Mianyang Central Hospital. Written informed consent was obtained from all patients. RA patients newly started on therapy in the department of rheumatology and immunology, Mianyang Central Hospital from March 2016 to March 2018 were recruited. Inclusion criteria were as follows: (1) meeting the RA diagnosis criteria (13); (2) all patients in active RA before treatment (DAS28 $\geq 2.6$ ), and achieving clinical remission after treatment (DAS28 $\leq 2.6$ ); (3) cooperation with the examinations and follow-up required for the study. Exclusion criteria were as follows: (1) joint disease caused by osteoporosis, trauma and other diseases; (2) autoimmune diseases such as systemic lupus erythematosus and Sjogren's syndrome; (3) pregnancy or lactation; (4) malignancy or infection.

\subsection{Collection of Demographic and Clinicobiologic Character- istics}

The demographic data of RA patients including age, gender, and duration were collected and the number of tender or swollen joints were recorded before treatment. Erythrocyte sedimentation rate (ESR) was analyzed using the Westergren method examined by Roller 20 (ALIFAX, Italy). DAS28 score was calculated by:

DAS28 $=0.56 \times \sqrt{ } \mathrm{TJC} 28+0.28 \times \sqrt{ } \mathrm{SJC} 28+0.7 \times \operatorname{Ln}(\mathrm{ESR})$ $+0.014 \times \sqrt{ } \mathrm{GH}$.
Where TJC28 refers to the number of tender joints of 28 counted, SJC28 to the number of swollen joints of 28 counted, and GH to general health status (14). Patients were divided into low-activity $(2.6<$ DAS28 $\leq 3.2)$, moderate-activity $(3.2<$ DAS2 $\leq 5.1)$, and severe-activity (DAS28 > 5.1) according to their DAS28 scores.

\subsection{Musculoskeletal Ultrasonography}

Musculoskeletal ultrasonography of the wrists, hands and forefeet was performed with Mindray Resona7 or Mindray DC-8 (Mindray Bio-Medical Electronics Co., Ltd, Shenzhen, Guangdong, China). The hand and forefoot, which were more clinically affected by tenderness or swelling were chosen and examined. This included the joints most likely to be affected by RA: the wrist, metacarpophalangeal (MCP) II and III, proximal interphalangeal (PIP) II and III, metatarsophalangeal (MTP) II and V joints. Each joint region was investigated in dorsal, ventral, and, when possible, lateral views in GSUS and CDUS. The scoring methods of synovitis by GSUS and CDUS, tenosynovitis by GSUS and CDUS and erosions are shown in Table 1 . The sum of these scores is US7 score (10). All RA patients underwent musculoskeletal ultrasonography to evaluate the US7 score before treatment and the beginning of follow-up.

\subsection{Clinical Treatment Protocol}

Patients in the low-activity and moderate-activity without poor prognostic factors received conventional diseasemodifying antirheumatic drugs (cDMARDs) as a starting treatment. Patients in the moderate-activity with poor prognostic factors and severe-activity received a combined treatment of two or three types of cDMARDs or biologic disease-modifying antirheumatic drugs (bDMARDs), such as tumor necrosis factor-alpha antagonist or interleukin6 antagonist. The DAS28 of each patient was re-evaluated monthly and the treatment protocol was adjusted every 3 months according to the DAS28. After clinical remission (DAS28 $\leq 2.6$ ), the DAS28 was re-evaluated monthly and the original treatment plan was maintained for at least 3 months. The treatment dose was gradually reduced when the clinical remission was maintained for 3 months. The successful treatment was defined when clinical remission was maintained for at least 6 months.

\subsection{Follow-Up}

A one-year follow-up was performed for all successful treated RA patients who had reached clinical remission and their US7 scores and DAS28 were recorded as the baseline indicators before the beginning of follow-up. DAS28 was evaluated every 3 months (3rd, 6th, 9th, and 12th 


\begin{tabular}{|c|c|c|c|c|}
\hline Score & Synovitis by GSUS & Tenosynovitis by GSUS & $\begin{array}{l}\text { Synovitis or tenosynovitis by } \\
\text { CDUS }\end{array}$ & Erosions \\
\hline $\mathbf{0}$ & Absence & No hypoechoic hyperplastic tissue & No intraarticular color signal & $\begin{array}{l}\text { Continuous echo of the bone } \\
\text { surface }\end{array}$ \\
\hline 1 & $\begin{array}{l}\text { A small hypoechoic/anechoic line } \\
\text { beneath the joint capsule }\end{array}$ & $\begin{array}{l}\text { A hypoechoic/anechoic thickened } \\
\text { tissue with/without fluid within the } \\
\text { tendon sheath }\end{array}$ & $\begin{array}{l}\text { Up to } 3 \text { color signals or } 2 \text { single and } \\
1 \text { confluent signal in the } \\
\text { intraarticular area }\end{array}$ & $\begin{array}{l}\text { An interruption of the bone surface } \\
\text { in } 2 \text { perpendicular planes }\end{array}$ \\
\hline 2 & $\begin{array}{l}\text { Joint capsule elevated parallel to } \\
\text { the joint area }\end{array}$ & & $\begin{array}{l}<50 \% \text { of the intraarticular area } \\
\text { filled with color signals }\end{array}$ & \\
\hline 3 & $\begin{array}{l}\text { A strong distension of the joint } \\
\text { capsule }\end{array}$ & & $\begin{array}{l}\geq 50 \% \text { of the intraarticular area } \\
\text { filled with color signals }\end{array}$ & \\
\hline
\end{tabular}

Abbreviations: CDUS, color Doppler ultrasound; GSUS, gray-scale ultrasound; US7 score, seven-joint ultrasonic score.

month) during the follow-up. Recurrence was defined if DAS28 $>2.6$ or the increase of DAS28 $>0.6$ compared to the baseline were found at any time during the follow-up. The patients were divided into the recurrence group and nonrecurrence group according to their recurrent results, and the clinical indicators and US7 scores before follow-up were compared and analyzed between the two groups (Figure1).

\subsection{Statistical Analysis}

Statistical Product and Service Solutions (version 22.0; SPSS Inc., Chicago, IL, USA) and Medcalc (Version 22.0.1; MedCalc Software, Ostend, Belgium) were used for statistical analysis. The numerical data were expressed as mean \pm standard deviations for normally distributed variables and as median (interquartile range) for other continuous variables. Independent sample $t$-tests and Mann-Whitney U-tests were used for comparison, and the paired variables were compared by Wilcoxon signed rank test. The categorical variables were expressed as number and the comparison was performed by the chi-square test. The association between US7 score and DAS28 before treatment was analyzed with Spearman correlations. The recurrence-free survival curves were plotted using Kaplan-Meier method and compared using the logrank statistic to analyze the difference in recurrence rate between RA patients with subclinical synovitis (US7 score $\neq 0$ ) and without subclinical synovitis (US7 score $=0$ ). Univariate COX regression analysis of US7 score was performed to analyze its effect on the recurrence of RA patients after treatment. Receiver-operating characteristics (ROC) curves were established to evaluate the predictive accuracy of the DAS 28 and US7 score for prognosis, and a combined prediction model was established by Logistic regression. Statistical significance was defined as two-tailed $\mathrm{P}<0.05$ for all tests.

\section{Results}

\subsection{Clinical Indicators and Ultrasonic Image Characteristics of RA Patients}

A total of 186 RA patients were included in this study (41 males and 145 females, mean age of $54.09 \pm 11.12$ years, median disease duration of 9 months, and median DAS28 of 5.2). Twenty patients were diagnosed as low-activity, and the gray-scale ultrasonic image showed mild synovial hyperplasia and Doppler blood flow signal was not obvious (Figure 2A). In 93 RA patients with moderate-activity, it showed a moderate synovial hyperplasia with a little blood flow signal (Figure 2B). In 73 RA patients with severeactivity, a severe synovial hyperplasia with significantly increased blood flow signal was displayed (Figure 2C). The ultrasonic features revealed that most RA patients had GSUS synovitis and CDUS synovitis. GSUS synovitis was more common in the wrist, MCP2 and MTP2, and CDUS synovitis was more common in the wrist and MTP. According to the US7 score, the median US7 score was 18. Correlation analysis showed that the US7 score was positively correlated with DAS28 before treatment $(\mathrm{r}=0.535, \mathrm{P}<0.001)$ (Figure 3 ).

\subsection{Comparison of the DAS28 and US7 Score Before and After Treatment}

Of the 186 RA patients, 104 (55.9\%) received cDMARDs and the remaining $82(44.1 \%)$ received bDMARDs. After successful treatment, DAS28, GSUS synovitis, CDUS synovitis, GSUS tenosynovitis, CDUS tenosynovitis, and US7 score of RA patients were significantly lower than those before treatment (all $\mathrm{P}<0.001$, Table 2).

4.3. Recurrence Analysis of One-Year Follow-Up for RA Patients with Disease in Remission

Totally, 60 RA patients suffered recurrence (recurrence rate $32.3 \%$ ) were included in the recurrence group, and 


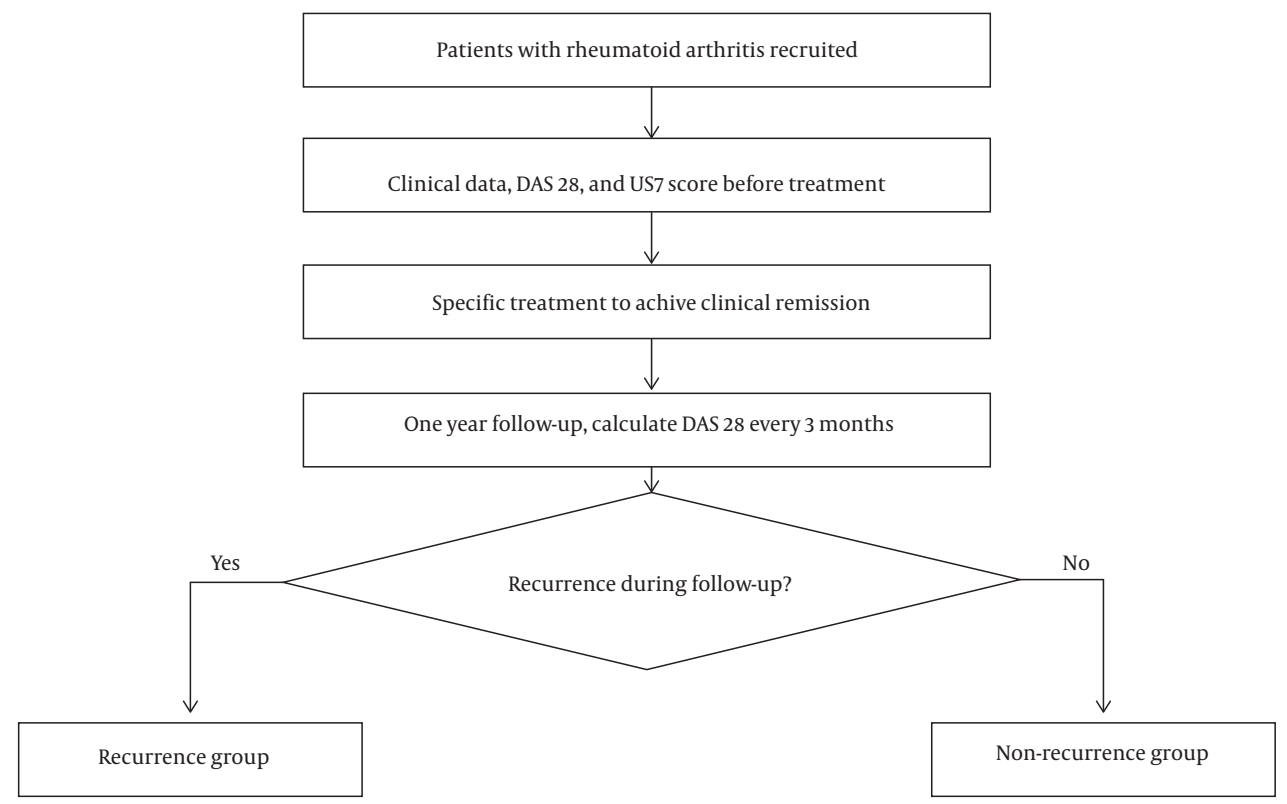

Figure 1. Flow chart of the recruitment, treatment, follow-up and grouping of RA patients. RA, rheumatoid arthritis; DAS28, disease activity score in 28 joints; US7 score, seven-joint ultrasonic score.
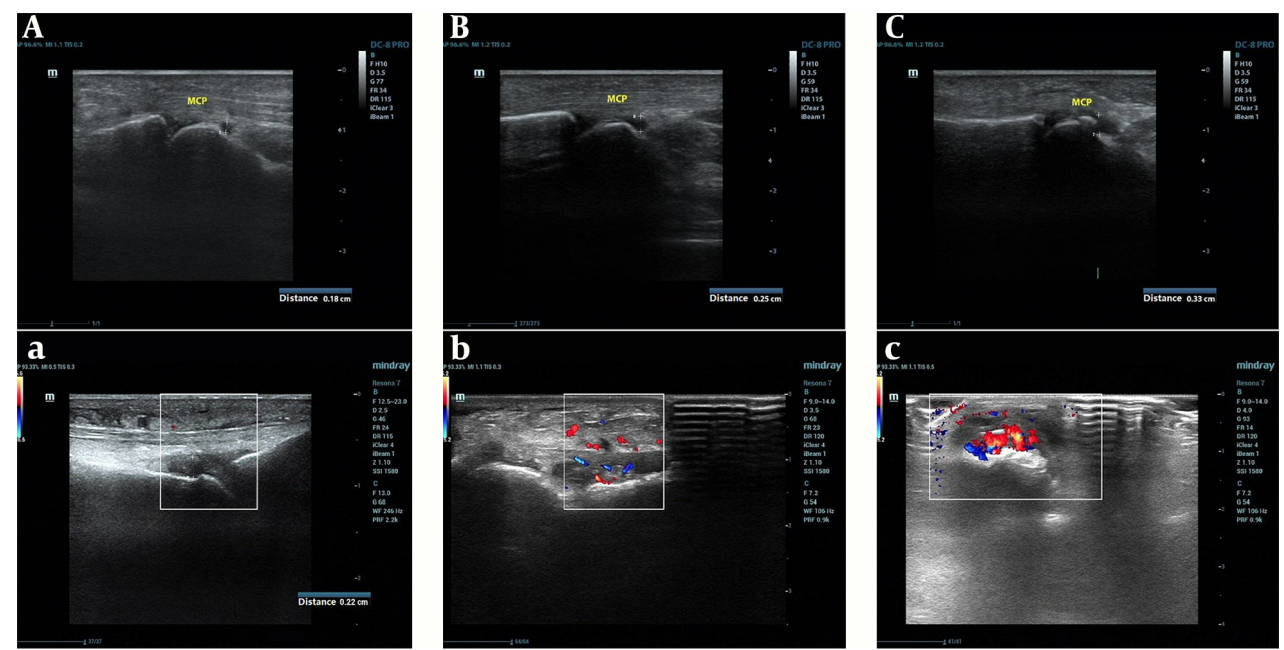

Figure 2. Ultrasonic evidence of RA patients in different activities. A, Gray-scale ultrasonic image of RA patients with low-activity. The image shows mild synovial fluid in MCP and GSUS is level 1. a, CDFI of RA patients with low-activity. No obvious blood flow signal is found, and PDUS is level 0. B, Gray-scale ultrasonic image of RA patients with moderate-activity. The image shows moderate synovial fluid in MCP and GSUS is level 2. b, CDFI RA patients with moderate-activity. Multiple dot-shaped blood flow signals are displayed, and CDUS is level 1. C, Gray-scale ultrasound image of RA patients with severe-activity. The image shows severe synovial fluid in MCP (0.33 cm) and GSUS is level 3. c, CDFI RA patients with severe-activity. Abundant blood flow signals are displayed, and CDUS is level 1. RA, rheumatoid arthritis; MCP, metacarpophalangeal; GSUS, gray-scale ultrasound; CDFI, color Doppler flow imaging; CDUS, Color Doppler ultrasound.

the remaining 126 patients were included in the nonrecurrence group. DAS28, US7 score, GSUS synovitis, and CDUS synovitis in the recurrence group were greater than those in the non-recurrence group $(\mathrm{P}<0.05$, Table 3$)$.
4.4. Influence of the Subclinical Synovitis on the Recurrence of RA Patients in Clinical Remission After Treatment

There were 78 RA patients without subclinical synovitis (US7 score $=0$ ) after successful treatment and eight of them recurred (recurrence rate 10.26\%). Totally, there were 108 


\begin{tabular}{|c|c|c|c|c|c|c|c|}
\hline & DAS28 & GSUS synovitis & CDUS synovitis & GSUS tenosynovitis & CDUS tenosynovitis & Erosions & US7 score \\
\hline Before treatment & $5.2(3.8,6.8)$ & $10(7,15.25)$ & $5(2,8)$ & $2(1,3)$ & $1(0,2)$ & $0(0,0)$ & $18(13,25)$ \\
\hline After treatment & $1.7(1.3,2.1)$ & $2(0,5)$ & $0(0,0)$ & $0(0,0)$ & $0(0,0)$ & $0(0,0)$ & $2(0,5)$ \\
\hline $\mathbf{P}$ & $<0.001$ & $<0.001$ & $<0.001$ & $<0.001$ & $<0.001$ & 1.000 & $<0.001$ \\
\hline
\end{tabular}

Abbreviations: CDUS, color Doppler ultrasound; DAS28, disease activity score in 28 joints; GSUS, gray-scale ultrasound; US7 score, seven-joint ultrasonic score.

${ }^{a}$ Statistical tests: Wilcoxon signed rank test for all data.

\begin{tabular}{|c|c|c|c|}
\hline & Recurrence group & Non-recurrence group & $\mathbf{P}$ \\
\hline Gender (Male/Female) & $13 / 47$ & $28 / 98$ & 0.932 \\
\hline Age, $y$ & $52.95 \pm 11.06$ & $54.63 \pm 11.15$ & 0.335 \\
\hline RA duration, mo & $8.57 \pm 3.97$ & $9.28 \pm 3.59$ & 0.224 \\
\hline Rheumatoid factor & $175.01 \pm 45.39$ & $179.34 \pm 53.4$ & 0.589 \\
\hline DAS28 after treatment & $1.8(1.2,2.2)$ & $1.45(1.1,1.8)$ & 0.009 \\
\hline US7 score after treatment & $4.5(2,7)$ & $0(0,2)$ & $<0.001$ \\
\hline GSUS synovitis & $4(2,6)$ & $0(0,2)$ & $<0.001$ \\
\hline CDUS synovitis & $0(0-3)$ & $0(0-1)$ & $<0.001$ \\
\hline GSUS tenosynovitis & $0(0-3)$ & $0(0-0)$ & 0.040 \\
\hline CDUS tenosynovitis & $0(0-1)$ & $0(0-1)$ & 0.200 \\
\hline Erosions & $0(0-2)$ & $0(0-1)$ & 0.962 \\
\hline
\end{tabular}

Abbreviations: CDUS, color Doppler ultrasound; DAS28, disease activity score in 28 joints; GSUS, gray-scale ultrasound; US7 score, seven-joint ultrasonic score.

${ }^{\mathrm{a}}$ Values are expressed as mean $\pm \mathrm{SD}$.

${ }^{b}$ Statistical tests: chi-square test for gender; students' $t$-test for age, RA duration, and rheumatoid factor; Mann-Whitney U-test for DAS28 after treatment, US7 score after treatment, GSUS synovitis, CDUS synovitis, GSUS tenosynovitis, CDUS tenosynovitis, and erosions.

RA patients with subclinical synovitis (US7 score $\neq 0$ ) after successful treatment, of which 52 recurred (recurrence

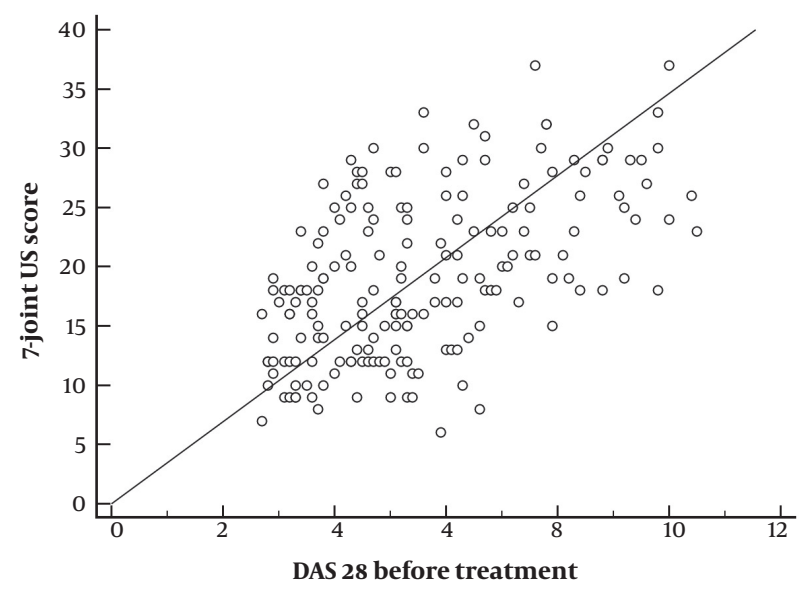

Figure 3. Scatter plot of the correlation between the US7 score and DAS28 before treatment. US7 score, seven-joint ultrasonic score; DAS28, disease activity score in 28 joints. rate $48.15 \%$, which was higher than those without subclinical synovitis (Logrank $\chi^{2}=27.556, \mathrm{P}<0.001$, Figure 4$)$. Univariate COX regression analysis found that the US7 score was associated with an increased risk of recurrence in RA patients after successful treatment $(\mathrm{P}<0.001)$. Each point increase in US7 score was associated with an average $36 \%$ increase in the risk of recurrence (hazard ratio $=1.363,95 \%$ $\mathrm{CI}=1.247-1.488)$.

4.5. Accuracy of US7 Score and DAS28 for Predicting the Prognosis of RA Patients

The area under the curve (AUC) of US7 score (0.808) was higher than DAS28 (AUC $=0.619$ ), with a sensitivity and specificity of $70.00 \%$ and $76.19 \%$, respectively. The AUC of the combined prediction model of US7 score and DAS 28 (0.904) was higher than that of the US7 score and DAS28 $(\mathrm{P}<0.05)$, with a sensitivity and specificity of $85.00 \%$ and 79.37\%, respectively (Figure 5 and Table 4 ). 


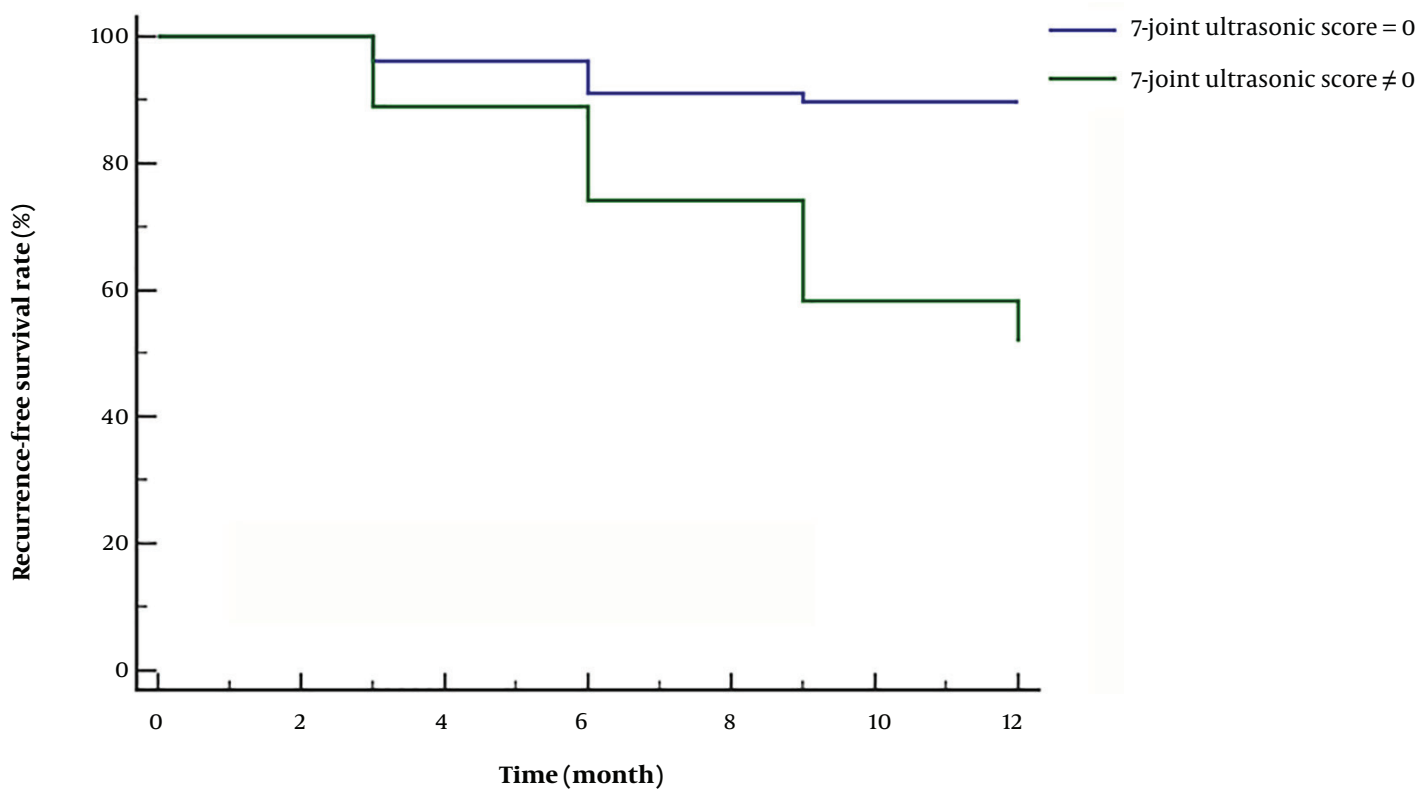

Figure 4. Kaplan-Meier analysis of the effect of US7 score on the outcome of RA patients. The recurrence rate of RA patients with subclinical synovitis (US7 score $\neq 0$ ) after successful treatment was higher than those without subclinical synovitis (US7 score $=0$ ) $\left(\right.$ Logrank $\left.\chi^{2}=27.556, P<0.001\right)$. US7 score, seven-joint ultrasonic score; RA, rheumatoid arthritis.

Table 4. Accuracy of US7 Score and DAS28 for Predicting the Prognosis of RA Patients ${ }^{\mathrm{a}}$

\begin{tabular}{lcccc}
\hline & AUC & 95\% CI & Cut off point & Sensitivity, \% \\
\hline US7 score & 0.808 & $0.744-0.862$ & 2 & 70.00 \\
DAS28 & 0.619 & $0.545-0.689$ & 1.9 & 46.67 \\
Combination & 0.904 & $0.852-0.942$ & 0.35 & 84.92 \\
\hline
\end{tabular}

Abbreviations: AUC, area under curve; 95\% CI, 95\% confidence interval; DAS28, disease activity score in 28 joints; US7 score, seven-joint ultrasonic score.

${ }^{\mathrm{a}}$ US7 score vs DAS 28: $\mathrm{z}=3.296, \mathrm{P}=0.001$. Combination vs US7 score: $\mathrm{z}=4.280, \mathrm{P}<0.001$.

\section{Discussion}

In the present study, the US7 score was positively correlated with DAS28, which indicated a high application value in the assessment of RA disease activity, similar to DAS28. Moreover, the disease activity could be assessed more intuitively and objectively by ultrasonography. RA patients with low-activity showed mild synovial hyperplasia and no Doppler blood flow signal. Synovial hyperplasia is obvious in RA patients with moderate-to-severe activity and is accompanied by significant blood flow signals. It suggested that in addition to the assessment of different disease activities, ultrasound examination is helpful to clinical treatment adjustment by imaging characteristics.

The recurrence rate of RA patients with disease in clinical remission within 1 - 2 years was $31 \%$ - 52\% (15). In this study, the recurrence rate of RA patients in clinical remission was 32.3\%. It suggested that patients' joints would con- tinue to be damaged even if they have achieved clinical remission due to the indiscoverable subclinical synovitis. Although the study conducted by Nguyen et al. (16) proposed that the US7 score is helpful for the discovery of subclinical synovitis in RA patients, to our knowledge, there are few related studies to prove its prediction value for the recurrence of RA patients.

Since US7 score $=0$ is the easiest method to determine whether there is subclinical synovitis in RA patients, this study analyzed the difference in recurrence between RA patients with and without subclinical synovitis. It showed that the recurrence rate of patients without subclinical synovitis (10.26\%) was significantly lower than that of patients with subclinical synovitis (48.15\%). It suggested that the US7 score $\neq 0$ might be a simple indicator to predict recurrence after, clinical remission. However, we must be aware that this is not an accurate method because it will increase the false-positive rate and would only rely on a 


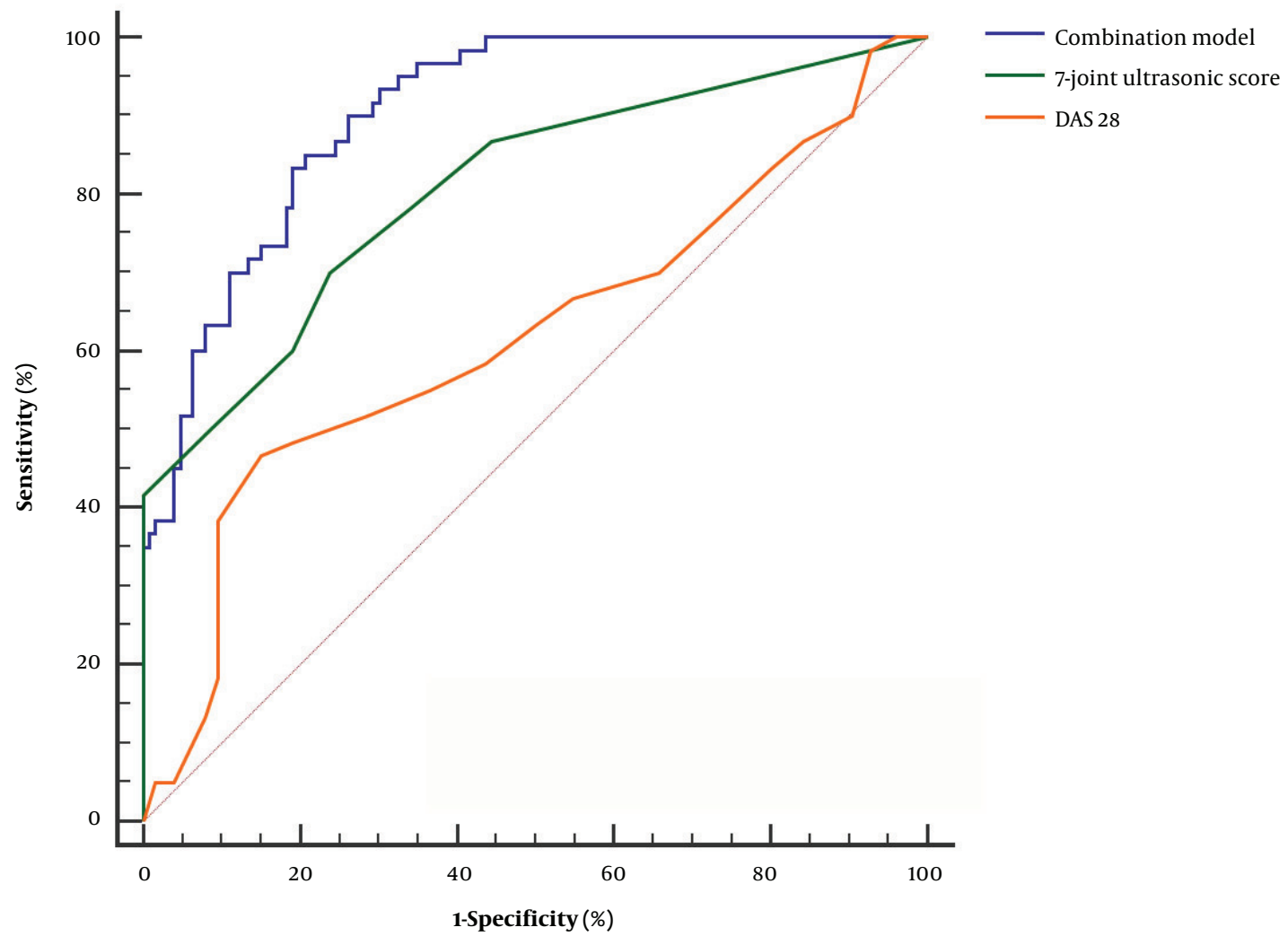

Figure 5. ROC curves of US7 score and DAS28 in predicting the recurrence of RA patients in clinical remission. US7 score, seven-joint ultrasonic score; DAS28, disease activity score in 28 joints; RA, rheumatoid arthritis; ROC, receiver-operating characteristics.

US7 score $=0$. There is also a physiological effusion in the peripheral joint cavity of healthy people, especially loadbearing joints, and these fluids can also be detected by ultrasound examination (17).

In the present study, the ROC curve showed that the AUC of US7 score was below 0.9 although it was higher than DAS28. It indicated that the US7 score alone was not accurate, and the combination was needed to improve the predictive accuracy. The combined prediction model of US7 score and DAS 28 showed a higher AUC (0.909), suggesting that the combined model could accurately predict the outcome of RA patients in clinical remission. Physicians may record the US7 score and DAS28 of RA patients and predict their probability of recurrence using this model to adjust clinical treatment.

RA patients in clinical remission are easily to recur due to subclinical synovitis. The results of our study are helpful to predict the recurrence risk of such patients. However, our study is a single-center study and the sample size is limited, there may be bias in the results, and a large sample size is needed for further verification. This study found that the accuracy of the combined prediction model is better than that of DAS28 or US7 score alone. It is believed that with larger sample sizes, we could predict the recurrence of RA patients more accurately.

In conclusion, the US7 score could predict the recurrence of RA patients more accurately because of its advantages in identifying subclinical synovitis. The combined prediction model of DAS28 and US7 score was the most accurate in predicting the recurrence of RA patients with disease in clinical remission.

\section{Acknowledgments}

The authors wish to thank all the study participants, research staff and students who participated in this work.

\section{Footnotes}

Authors' Contributions: Study design: Xiao-Pan Zou, JinMei Zou, and Dao-ning Guo. Data collection and analysis: 
Dao-Ning Guo, Jia-Gao Qi, Yue Li, and Gang Chen. Supervision: Xiao-pan Zou and Jin-Mei Zou. Statistics: Xiao-Pan Zou, Jia-Gao Qi, Yue Li, and Gang Chen. Manuscript writing: Xiao-Pan Zou, Jin-Mei Zou, Dao-ning Guo, Jia-Gao Qi1, Yue Li1, and Gang Chen. Manuscript revision: Xiao-Pan Zou and Jin-Mei Zou. Approval of the manuscript: all authors.

Conflict of Interests: The authors declare that they have no conflict of interest.

Ethical Approval: Ethical approval for the study was obtained from the Ethics Committee of Mianyang Central Hospital (no.: P2020013).

Funding/Support: This study was supported by the first batch of Sichuan science and technology plan in 2017 (no.: 2017SZ0148).

Informed Consent: Written informed consent was obtained from all patients.

\section{References}

1. Terao C, Brynedal B, Chen Z, Jiang X, Westerlind H, Hansson M, et al. Distinct HLA associations with rheumatoid arthritis subsets defined by serological subphenotype. Am J Hum Genet. 2019;105(4):880. doi: 10.1016/j.ajhg.2019.09.018. [PubMed: 31585111]. [PubMed Central: PMC6817550].

2. Silva Luna K, Ortiz AM, Patino E, Aguilera C, Velasco T, Garcia de Vicuna $\mathrm{R}$, et al. Influence of the structure of mood in the assessment of rheumatoid arthritis through the visual analog scale for pain, HAQ and DAS28. Reumatol Clin. 2012;8(6):328-33. doi: 10.1016/j.reuma.2012.04.002. [PubMed: 23022221].

3. Peluso G, Michelutti A, Bosello S, Gremese E, Tolusso B, Ferraccioli G. Clinical and ultrasonographic remission determines different chances of relapse in early and long standing rheumatoid arthritis. Ann Rheum Dis. 2011;70(1):172-5. doi:10.1136/ard.2010.129924. [PubMed: 21097799].

4. Fayet F, Savel C, Rodere M, Pereira B, Abdi D, Mathieu S, et al. The development of a questionnaire to evaluate rheumatoid arthritis patient's knowledge about methotrexate. J Clin Nurs. 2016;25(5-6):682-9. doi: 10.1111/jocn.12999. [PubMed: 26526669].

5. Zavada J, Hanova P, Hurnakova J, Szczukova L, Uher M, Forejtova $S$, et al. The relationship between synovitis quantified by an ultrasound 7-joint inflammation score and physical disability in rheumatoid arthritis - a cohort study. Arthritis Res Ther. 2017;19(1):5. doi: 10.1186/s13075-016-1208-6. [PubMed: 28086960]. [PubMed Central: PMC5237153].

6. Barber CEH, Schieir O, Lacaille D, Marshall DA, Barnabe C, Hazlewood $\mathrm{G}$, et al. High adherence to system-level performance measures for rheumatoid arthritis in a national early arthritis co- hort over eight years. Arthritis Care Res (Hoboken). 2018;70(6):84250. doi: 10.1002/acr.23439. [PubMed: 29450976]. [PubMed Central: PMC6001563].

7. Inamo J, Kaneko Y, Sakata K, Takeuchi T. Impact of subclinical synovitis in ankles and feet detected by ultrasonography in patients with rheumatoid arthritis. Int J Rheum Dis. 2019;22(1):62-7. doi: 10.1111/1756185X.13399. [PubMed: 30338640].

8. Son KM, Lee SY, Seo YI, Choi JE, Kim HA. Contribution of subjective Disease Activity Score 28 (DAS28) components to the response to treatment of rheumatoid arthritis. Clin Rheumatol. 2017;36(6):1221-7. doi: 10.1007/s10067-017-3628-3. [PubMed: 28451869].

9. Burgers LE, Ten Brinck RM, van der Helm-van Mil AHM. Is joint pain in patients with arthralgia suspicious for progression to rheumatoid arthritis explained by subclinical inflammation? A crosssectional MRI study. Rheumatology (Oxford). 2019;58(1):86-93. doi: 10.1093/rheumatology/key220. [PubMed: 30137540]. [PubMed Central: PMC6319603].

10. Backhaus M, Ohrndorf S, Kellner H, Strunk J, Backhaus TM, Hartung W, et al. Evaluation of a novel 7-joint ultrasound score in daily rheumatologic practice: A pilot project. Arthritis Rheum. 2009;61(9):1194-201. doi: 10.1002/art.24646. [PubMed: 19714611].

11. Zhou L, Song J, Chen L, Xu HJ. [The application of matrix metalloproteinase- 3 and 7 joints ultrasonic score in assessment of disease activity in patients with rheumatoid arthritis]. Zhonghua Nei Ke Za Zhi. 2016;55(7):531-4. Chinese. doi: 10.3760/cma.j.issn.05781426.2016.07.009. [PubMed: 27373288].

12. Sapundzhieva T, Karalilova R, Batalov A. Musculoskeletal ultrasound for predicting remission in patients with rheumatoid arthritis: Results from a 1-year prospective study. Rheumatol Int. 2018;38(10):1891-9. doi: 10.1007/s00296-018-4131-0. [PubMed:30121699].

13. Aletaha D, Neogi T, Silman AJ, Funovits J, Felson DT, Bingham C3, et al. 2010 rheumatoid arthritis classification criteria: An American College of Rheumatology/European League Against Rheumatism collaborative initiative. Ann Rheum Dis. 2010;69(9):1580-8. doi: 10.1136/ard.2010.138461. [PubMed: 20699241].

14. van Riel PL. The development of the disease activity score (DAS) and the disease activity score using 28 joint counts (DAS28). Clin Exp Rheumatol. 2014;32(5 Suppl 85):S-65-74. [PubMed: 25365092].

15. Geng Y, Han J, Deng X, Zhang Z. Presence of power Doppler synovitis in rheumatoid arthritis patients with synthetic and/or biological disease-modifying anti-rheumatic drug-induced clinical remission: Experience from a Chinese cohort. Clin Rheumatol. 2014;33(8):1061-6. doi: 10.1007/s10067-014-2634-y. [PubMed: 24777468].

16. Nguyen H, Ruyssen-Witrand A, Gandjbakhch F, Constantin A, Foltz V, Cantagrel A. Prevalence of ultrasound-detected residual synovitis and risk of relapse and structural progression in rheumatoid arthritis patients in clinical remission: A systematic review and meta-analysis Rheumatology (Oxford). 2014;53(11):2110-8. doi: 10.1093/rheumatology/keu217. [PubMed: 24929634].

17. El Miedany Y. Musculoskeletal US: Examining the joints. $\mathrm{Br} J$ Nurs. 2012;21(6):340-4. doi: 10.12968/bjon.2012.21.6.340. [PubMed: 22584929]. 